\title{
Gas-kinetic scheme for rarefied flow simulation
}

\author{
Kun $\mathrm{Xu}^{\mathrm{a}, *}$, Eswar Josyula ${ }^{\mathrm{b}}$ \\ a Mathematics Department, Hong Kong University of Science and Technology, Hong Kong \\ ${ }^{\mathrm{b}}$ Air Force Research Laboratory, 2210 Eighth Street Wright-Patterson AFB, OH 45433, USA
}

Available online 10 July 2006

\begin{abstract}
For increasingly rarefied flowfields, the predictions from continuum formulation, such as the Navier-Stokes equations lose accuracy. These inaccuracies are attributed primarily to the linear approximations of the stress and heat flux terms in the NavierStokes equations. The inclusion of higher order terms, such as Burnett, or high-order moment equations, could improve the predictive capabilities of such continuum formulations, but there has been limited success in the shock structure calculations, especially in the high Mach number case. Here, after reformulating the viscosity and heat conduction coefficients appropriate for the rarefied flow regime, we will show that the extended Navier-Stokes-type continuum formulation may still be properly used. The equations with generalized dissipative coefficients based on the closed solution of the Bhatnagar-Gross-Krook (BGK) model of the Boltzmann equation, are solved using the gas-kinetic numerical scheme.
\end{abstract}

(C) 2006 IMACS. Published by Elsevier B.V. All rights reserved.

Keywords: Rarefied flow; Shock structure; BGK model

\section{Introduction}

The classification of the various flow regimes based on the dimensionless parameter, the Knudsen number, is a measure of the degree of rarefaction of the medium. The Knudsen number $K n$ is defined as the ratio of the mean free path to a characteristic length scale of the system. In the continuum flow regime where $K n \leq 0.01$, the Navier-Stokes equations with linear relations between stress and strain and the Fourier's law for heat conduction are adequate to model the fluid behavior. For flows in the continuum-transition regime $(0.1<K n<1)$, the Navier-Stokes equations are known to be inadequate. This regime is important for many practical engineering problems, such as the simulation of microscale flows and hypersonic flow around space vehicles in low earth orbit. Hence, there is a strong desire and requirement for accurate models which give reliable solutions with lower computational costs. Among continuum solution methodologies, there are primarily two approaches: the Chapman-Enskog method, and the method of moments [2]. In the Chapman-Enskog method, the phase density is expanded in powers of the Knudsen number, the zeroth order expansion yielding the Euler equations, the first-order resulting in the equations of Navier-Stokes and Fourier, the second order the Burnett equations, and the third order expansion the so-called super-Burnett equations. It is well recognized that the equations of Navier-Stokes and Fourier cease to be accurate for Knudsen number above 0.1. Unfortunately, the higher order equations are shown to be linearly unstable for processes involving small wavelengths,

\footnotetext{
* Corresponding author.

E-mail address: makxu@ust.hk (K.Xu).
} 
or high frequencies, and thus cannot be used in numerical simulations. The goal of this study is to present a continuum formulation for low density rarefied gas flows. The continuum model developed in the present study will be used to solve non-equilibrium shock structures for monatomic and diatomic gases.

\section{Non-equilibrium continuum model}

The Boltzmann equation expresses the behavior of a many-particle kinetic system in terms of the evolution equation for a single particle gas distribution function. The simplification of the Boltzmann equation given by the BGK model is formulated as

$$
\frac{\partial f}{\partial t}+\mathbf{u} \frac{\partial f}{\partial \mathbf{x}}=\frac{f^{\mathrm{eq}}-f}{\tau},
$$

where $f$ is the number density of molecules at position $\mathbf{x}$ and particle velocity $\mathbf{u}$ at time $t$. The left hand side of the above equation represents the free streaming of molecules in space, and the right side denotes the collision term. If the distribution function $f$ is known, macroscopic variables, such as mass, momentum, energy and stress, can be obtained by integration over the moments of molecular velocity. Using Chapman-Enskog expansion, the Navier-Stokes equations for both monatomic and diatomic gases can be derived. It is well recognized that the standard form of Navier-Stokes equations with the bulk viscosity given by the Stokes hypothesis, cannot properly describe the non-equilibrium flow. In the general case of non-equilibrium, temperatures for the translational and rotational energy modes will be different. Here, we will discuss implementation of the non-equilibrium rotational relaxation model into the BGK model. The modelling of translational non-equilibrium through the generalization of particle collision time will be presented later.

In general, based on the above-mentioned BGK model, for a diatomic gas we assume two temperatures, one for translational and the other for rotational,

$$
f^{\mathrm{eq}}=\rho\left(\frac{\lambda_{\mathrm{t}}}{\pi}\right)^{3 / 2}\left(\frac{\lambda_{\mathrm{r}}}{\pi}\right) \mathrm{e}^{-\lambda_{\mathrm{t}}\left((u-U)^{2}+\xi^{2}\right)-\lambda_{\mathrm{r}} \eta^{2}},
$$

where $\lambda_{\mathrm{t}}=m / 2 k T_{\mathrm{t}}$ is related to the translational temperature $T_{\mathrm{t}}$, and $\lambda_{\mathrm{r}}=m / 2 k T_{\mathrm{r}}$ to the rotational temperature $T_{\mathrm{r}}$. The nitrogen molecule has two rotational degrees of freedom $\eta^{2}=\eta_{1}^{2}+\eta_{2}^{2}$, and $\xi^{2}=\xi_{1}^{2}+\xi_{2}^{2}$ is to account for the random translational motion of the particle in $y$ - and $z$-directions. The relation between mass, momentum, total energy, and rotational energy densities $\rho E_{\mathrm{r}}$ with the distribution function $f$ is

$$
W=\left(\rho, \rho U, \rho E, \rho E_{\mathrm{r}}\right)^{\mathrm{T}}=\int \psi f \mathrm{~d} u \mathrm{~d} \xi \mathrm{d} \eta,
$$

where $\psi$ has the components $\psi=\left(1, u, 1 / 2\left(u^{2}+\xi^{2}+\eta^{2}\right), 1 / 2 \eta^{2}\right)$. Only the mass, momentum and total energy are conserved during particle collisions, so $f$ and $f^{\text {eq }}$ satisfy the condition $\int\left(f^{\text {eq }}-f\right) \psi \mathrm{d} u \mathrm{~d} \xi \mathrm{d} \eta=S=(0,0,0, s)^{\mathrm{T}}$. From the BGK model, the source term $s$ for the rotational energy is given by, $s=\rho\left(E_{\mathrm{r}}^{\mathrm{eq}}-E_{\mathrm{r}}\right) / Z_{\mathrm{r}} \tau$. Here $\tau$ is the relaxation time for rotational energy to reach equilibrium, which has been generalized using the Landau-Teller-type model, sometimes referred to as the Jeans equation, to account for the longer relaxation time for the rotational non-equilibrium through the rotational collision number $Z_{\mathrm{r}}$. The equilibrium energy $\rho E_{\mathrm{r}}^{\mathrm{eq}}$ in the above equation is determined by

$$
T_{\mathrm{t}}=T_{\mathrm{r}}, \quad \rho E_{\mathrm{r}}^{\mathrm{eq}}=\frac{\rho}{2 \lambda^{\mathrm{eq}}}, \quad \text { and } \quad \lambda^{\mathrm{eq}}=\frac{5 \rho}{4\left(\rho E-0.5 \rho U^{2}\right)} .
$$

When the characteristic time for rotational relaxation is much longer than that of translational in the shock layer where the characteristic flow time is of the order of particle collision time, the Landau-Teller-Jeans model was used to account for the relaxation of rotational energy. For the translation temperature below $1400 \mathrm{~K}$ in a nitrogen gas which is of interest here, the use of a single rotational temperature and the Landau-Teller-Jeans model for rotational relaxation is assumed to be adequate in the present study. Then, the source term is defined as $S=\left(0,0,0, \frac{3 \rho}{Z_{\mathrm{r}} \tau}\left(T_{\mathrm{t}}-T_{\mathrm{r}}\right)\right)$, and the value $Z_{\mathrm{r}}$ for a certain diatomic molecule depends on the temperature. The collision time $\tau$ multiplied by a rotational collision number $Z_{\mathrm{r}}$ models the relaxation process for the rotational energy to equilibrate with the translational energy. For nitrogen gas, absorption measurements of ultrasonic sound yielded a value of $Z_{\mathrm{r}}$ equal to 5.26 and 5.5, while shock wave measurements using the electron-beam fluorescence technique have yielded $Z_{\mathrm{r}}=5$ at the room temperature. In the limit of small departure from equilibrium, we have, $T_{\mathrm{t}}-T_{\mathrm{r}}=-\frac{2}{3} \tau T U_{x}$, and the bulk viscosity term can be 
recovered $-\frac{2}{5} \rho R\left(T_{\mathrm{t}}-T_{\mathrm{r}}\right)=\frac{4}{15} \tau p U_{x}$. In the non-equilibrium case, the bulk viscosity effect has to be represented physically by the temperature relaxation between the translational and rotational temperature. In order to capture the non-equilibrium effect in the rarefied gas flow, the particle collision time has to be generalized. Our present model is based on the existence of the closed solution of the BGK model, which is assumed to be $f=f^{\mathrm{eq}}-\tau^{*}\left(f_{\mathrm{t}}^{\mathrm{eq}}+u f_{x}^{\mathrm{eq}}\right)$, where $\tau^{*}$ is the parameter to be determined. Substituting the above equation into the BGK model (1), we can obtain a relation between the generalized particle collision time $\tau^{*}$ and the collision time $\tau$, which is well-defined in the continuum flow regime through $\tau=\mu / p, \tau^{*}=\tau /\left(1+\tau D^{2} f^{\mathrm{eq}} / D f^{\mathrm{eq}}\right)$, where $D=\partial / \partial t+u \partial / \partial x$. In order to remove the dependence of the collision time $\tau^{*}$ on the individual molecular velocity, $\tau D^{2} f^{\text {eq }} / D f^{\text {eq }}$ can be evaluated by taking moment $\phi$, as $\int \phi D^{2} f^{\mathrm{eq}} \mathrm{d} u \mathrm{~d} \xi \mathrm{d} \eta / \int \phi D f^{\mathrm{eq}} \mathrm{d} u \mathrm{~d} \xi \mathrm{d} \eta$. Since, the stress and heat conduction terms are resulting from the moments of the gas distribution function, the value of $\tau^{*}$ in the viscosity term $\tau^{*} p$ and heat conduction coefficient $\tau^{*} p C_{p} / \operatorname{Pr}$ are obtained separately from different moments; $\phi_{1}=(u-U)^{2}$ for the viscosity coefficient and $\phi_{2}=(u-U)\left[(u-U)^{2}+\xi^{2}\right]$ for the heat conduction coefficient. The above developed continuum model is solved by the gas-kinetic BGK-NS scheme [4], where the numerical fluxes at cell interfaces are evaluated based on the gas distribution function,

$$
f=f^{\mathrm{eq}}-\tau^{*}\left(\frac{\partial f^{\mathrm{eq}}}{\partial t}+\mathbf{u} \frac{\partial f^{\mathrm{eq}}}{\partial \mathbf{x}}\right)+t \frac{\partial f^{\mathrm{eq}}}{\partial t} .
$$

\section{Non-equilibrium shock structures and conclusion}

One of the simplest and most fundamental gas dynamic phenomena that can be used for the model validation is the internal structure of a normal shock wave. Firstly, the shock wave represents a flow condition that is far from thermodynamic equilibrium, and secondly shock wave phenomena is unique in that it allows one to separate the continuum differential equations of fluid motion from the boundary conditions that would be required to complete a well-posed problem. The boundary conditions for a shock wave are simply determined by the Rankine-Hugoniot relations. Firstly, we present test cases on the shock structure for a monatomic gas. Comparisons of our results are made with the theoretical solution of the full Boltzmann equation obtained by Ohwada for the hard sphere molecules at a Mach number $M=3$ [3]. For the hard sphere molecules, the viscosity coefficient $\tau \sim \mu \sim T^{0.5}$, where the $x$ coordinate is normalized by $\sqrt{\pi} l_{0} / 2$ and $l_{0}$ is the mean free path of the gas molecules at the upstream condition. Fig. 1 shows the density and temperature of an argon shock structure for Mach number $M=3$. For the nitrogen shock wave, the DSMC method is known to provide accurate separation distance in the translational and rotational temperature profiles. With the same parameters as used in the DSMC computation [1] and using the Sutherland's law for viscosity, the shock structure for nitrogen gas at Mach number $M=11$ is simulated by the current method and the results are

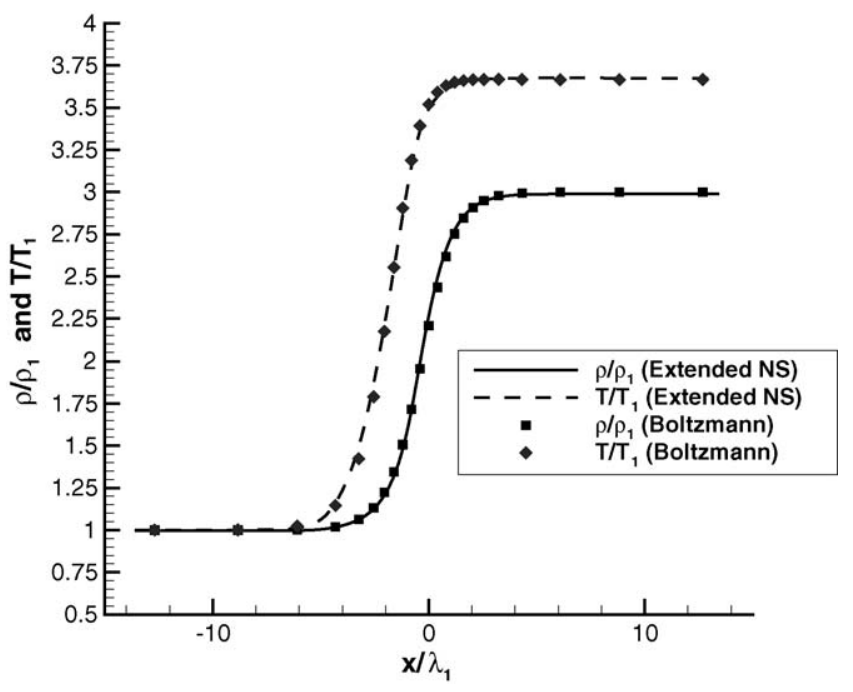

Fig. 1. $M=3.0$ argon shock structure for hard sphere gas. Boltzmann solution [3] vs. extended continuum model. 


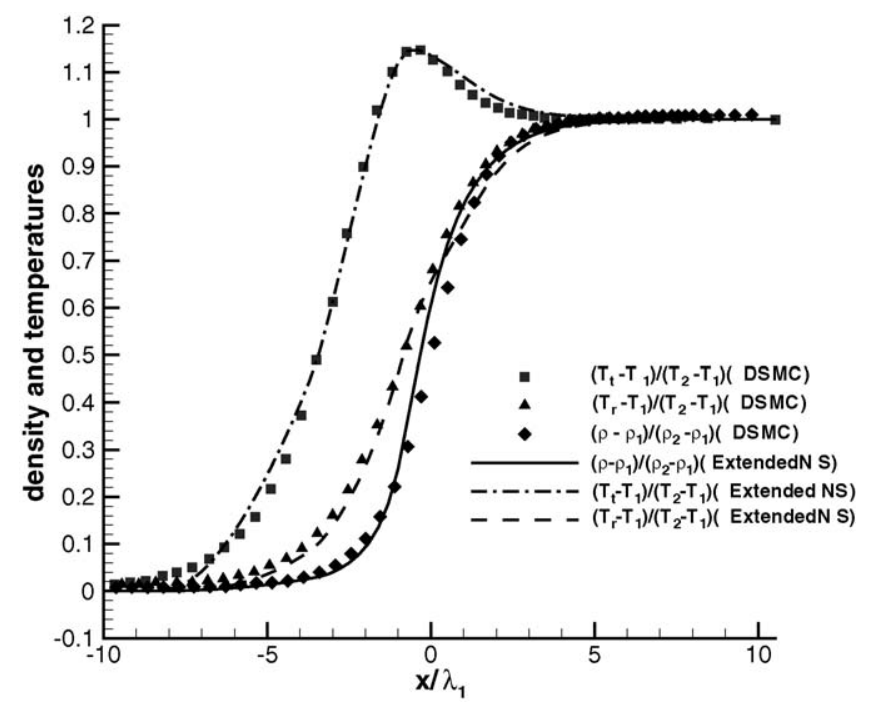

Fig. 2. $M=11$ nitrogen shock structure. DSMC solution [1] vs. extended continuum model.

shown in Fig. 2. The current extended NS continuum model reproduces the DSMC solution accurately. However, the continuum model only takes a few minutes to get the solution.

In conclusion, a continuum gas-kinetic formulation for the translational and rotational non-equilibrium flow is constructed. Based on the generalized constitutive relationships through the modification of particle collision time, the kinetic formulation truncated up to the Navier-Stokes order has been used in the shock structure computation. The new formulation is applied to both monatomic and diatomic shock structure calculations.

\section{Acknowledgements}

This research was supported by Hong Kong Research Grant Council HKUST6102/04E and 6210/05E and by U.S. Air Force Office of Scientific Research contracts monitored by F. Fahroo and J. Schmisseur.

\section{References}

[1] G.A. Bird, Aspects of the structure of strong shock waves, Phys. Fluids 13 (1970) 1172.

[2] S. Chapman, T.G. Cowling, The Mathematical Theory of Non-uniform Gases, Cambridge University Press, 1990.

[3] T. Ohwada, Structure of normal shock waves, Phys. Fluids A 5 (1993) 217.

[4] K. Xu, A gas-kinetic BGK scheme for the Navier-Stokes equations and its connection with artificial dissipation and Godunov method, J. Comput. Phys. 171 (2001) 289-335. 